\title{
Biomanagement of Meloidogyne Species in Bitter Gourd
}

\author{
Tulika Singh*, A. D. Patel, Anjana Prajapati and B. A. Patel \\ Department of Nematology, Anand Agriculture University, Anand- 388 110, India \\ *Corresponding author
}

\begin{tabular}{|c|}
\hline Keywords \\
\hline $\begin{array}{l}\text { Meloidogyne } \\
\text { incognita, } \\
\text { Purpureocillium } \\
\text { lilacium, } \\
\text { Pochoniachlamydos } \\
\text { poria }\end{array}$ \\
\hline Article Info \\
\hline $\begin{array}{l}\text { Accepted: } \\
\text { 07 September } 2019 \\
\text { Available Online: } \\
10 \text { October } 2019\end{array}$ \\
\hline
\end{tabular}

\section{A B S T R A C T}

The nematicidal effect of Purpureocillium lilacium, Pochonia chlamydosporia, Pseudomonas fluorescens singly or in combination were tested against root-knot nematodes under open field conditions. In the present study, the efficacy of four biocontrol agents was tested against Meloidogyne spp. The results revealed that soil application of vermicompost @2.5 tons/ha enriched with $P$. lilacinum @ $2.5 \mathrm{~kg}+$ vermicompost @ 2.5 tons of /ha enriched with P. fluorescens @ $2.5 \mathrm{~kg} / \mathrm{ha}$ (T4) proved effective to manage root-knot nematode, Meloidogyne spp. and hence increased bitter gourd fruit yield as compared to other treatments. The experiment proved that application of different biocontrol agents $(P$. lilacinum and $P$. chlamydosporia and P.fluorescens) not only has a lethal effect on nematode, but also enhances the plant growth, supplying many nutritional elements and induce the systemic resistance in plants. Through this investigation we could standardize a strategy for the sustainable management of nematode in bitter gourd.

\section{Introduction}

Plant parasitic nematodes, particularly rootknot nematodes, are widely distributed and cause significant yield losses in a wide range of crops (Sasser and Freckman, 1986). Meloidogyne incognita is a limiting factor affecting production of vegetables including Bottle gourd in India. The loss to Indian agriculture is estimated at aboutRs. 210 crore annually (Jain et al., 2007). Since use of chemicals is prohibitive as well as hazardous, attention has now been directed towards use of biopesticides, Purpureocillium lilacium, Pseudomonas fluorescens, Trichoderma viride and Pochonia chlamydosporium has a good potential as biocontrol agent to manage rootknot nematode, $M$. incognita (Mane and Mahase, 2017). Nematode management through biocontrol agents is gaining importance in the new millennium as other measures have become less attractive to 
growers/farmers where economics demand specialization and intensification. In the last decade nematode management through biocontrol agents was in the forefront of research and development. Management of plant parasitic nematodes through ecofriendly means is the need of this era. In view of the increasing awareness about environment and demand of organic farming, the present study was initiated to investigate the efficacy of different bioagents against root-knot nematodes, on Bottle gourd under field conditions.

\section{Materials and Methods}

The experiment was carried out in root-knot nematodes (Meloidogyne incognita and $M$. javanica) infested field at the initial population density ranging from 214-242 $\mathrm{J}_{2}$ per $200 \mathrm{~g}$ of soil at Department of Nematology, B. A. College of Agriculture, Anand Agricultural University, Anand during Kharif 2016 to 2018. Treatment combinations of bionematicide were given in Table 1.

Thus making a total of ten treatments including control was practiced in a Randomized Block Design with three replications. Bitter gourd variety Aakash was sown with $100 \times 150 \mathrm{~cm}$ spacing in $3.0 \times 7.5 \mathrm{~m}$ plot size. Vermicompost alone, vermicompost enriched with bio agents and FYM enriched with Bacillus pumilus were broadcasted in the plots and mixed with soil. Carbofuran was applied at the seeding spot and mixed with soil.

Then two seeds per spot were sown as per the recommended spacing. For treatment no. 7, after sowing plots were covered with green leaves of Sun hemp. All recommended agronomical practices were followed during experimentation. Observations on bitter gourd fruit yield at every picking, root-knot index at harvest and final soil and root nematode population were recorded and the data were subjected to statistical analysis.

\section{Results and Discussion}

The year wise data on efficacy of bio agents against Meloidogyne spp. in bittergourd are presented in Table 2, 3 and 4 while pooled over years data are presented in Table 5.

During the first year (2016), bitter gourd fruit yield was significantly higher in the treatment of soil application of Purpureocillium lilacinum@2.5 kg along with 2.5 tons of vermicompost/ha + Pseudomonas fluorescens @ $2.5 \mathrm{~kg} / \mathrm{ha}$ along with 2.5 tons of vermicompost/ha $\left(\mathrm{T}_{4}\right)$ but statistically it did not differ with $\mathrm{T}_{1}$ and $\mathrm{T}_{5}$.

Root- knot index were noticed non significant. Final nematode population from soil was significantly less in the treatment of $\mathrm{T}_{4}$. However, it was at par with $\mathrm{T}_{1}$ and $\mathrm{T}_{2}$. Non significant differences were noticed among the treatments in final nematode population from roots (Table 2).

During the second year of experimentation (2017), maximum yield (10.483 t/ha) was recorded in $\mathrm{T}_{4}$ even though it did not differ significantly from $\mathrm{T}_{1}, \mathrm{~T}_{2}, \mathrm{~T}_{5}, \mathrm{~T}_{6}, \mathrm{~T}_{7}$ and $\mathrm{T}_{8}$. Soil application of $P$. lilacinum@ $2.5 \mathrm{~kg}$ along with 2.5 tons of vermicompost/ha $+P$. fluorescens @ $2.5 \mathrm{~kg} / \mathrm{ha}$ along with 2.5 tons of vermicompost/ha has significantly less rootknot index (1.74) as compared to other treatments. However, it remained at par with $\mathrm{T}_{1}$. Final nematode population from soil was found non significant among the treatments. Whereas nematode population in roots was lowest (2.558) in the treatment of $\mathrm{T}_{8}$. However, it remained at par with $\mathrm{T}_{3}, \mathrm{~T}_{4}$ and $\mathrm{T}_{5}$ (Table 3). In the year 2018, treatment $\mathrm{T}_{4}$ gave significantly higher yield $(8.38 \mathrm{t} / \mathrm{ha})$ as compared to rest of the treatments except $T_{1}$. Differences for root-knot index among 
different treatments were found non significant. Final nematode population in soil was significantly less (1.977) in the treatment $\mathrm{T}_{4}$ and it remained at par with $\mathrm{T}_{1}$.

While nematode population in root was lowest (2.566) in $\mathrm{T}_{4}$ even though statistically it was at par with $\mathrm{T}_{1}, \mathrm{~T}_{2}$ and $\mathrm{T}_{8}$ (Table 4).

Data were pooled at the end of three years (2016,17and 2018). Results shows that soil application of $P$. lilacinum (cfu 2x 106) @ 2.5 $\mathrm{kg} / \mathrm{ha}$ along with 2.5 tons of vermicompost/ha + P. fluorescens (cfu 2x 106) @ $2.5 \mathrm{~kg} / \mathrm{ha}\left(\mathrm{T}_{4}\right)$ gave significantly highest yield $(8.02 \mathrm{t} / \mathrm{ha})$, but it did not differ significantly from $T_{1}$ and $T_{5}$. In case of root-knot index, it was lowest (1.84) in $\mathrm{T}_{4}$ and was at par with $\mathrm{T}_{1}, \mathrm{~T}_{2}, \mathrm{~T}_{5}, \mathrm{~T}_{6}, \mathrm{~T}_{8}$ and $\mathrm{T}_{9}$. Soil nematode population at harvest, it was minimum in $\mathrm{T}_{4}$ followed by $\mathrm{T}_{1}$, both were at par with each other and also not differed significantly from $\mathrm{T}_{2}, \mathrm{~T}_{3}, \mathrm{~T}_{5}, \mathrm{~T}_{6}$ and $\mathrm{T}_{8}$ (Table $5)$.

On foregoing discussion and based on effective nematode control as well as yield the treatment comprising soil application of vermicompost@2.5 tons/ha enriched with $P$. lilacinum@2.5 kg + vermicompost@2.5 tons of /ha enriched with P. fluorescens @ 2.5 $\mathrm{kg} / \mathrm{ha}$ proved effective to manage root-knot nematode, Meloidogyne spp. and hence increased bitter gourd fruit yield as compared to other treatments. The best protection against the Meloidogyne spp. was observed on the integration of vermicompost, which resulted increased plant growth and reduced build up of nematode and root galling.

It may be indicated on the basis of findings by various workers that nematophagous fungi are capable of producing toxic substances in the presence of suitable substrate in rhizosphere of plants. The presence of high population of such fungi in the rhizosphere where nematode population is also high may help in reducing the deleterious effects of nematodes on plants by suppressing their population.

Several authors proved that efficacy of combinations of more than one bio-agent can provide greater protection against disease than that of a single bio-agent (Ehteshamul-Haque et al., 1995; Izhar et al., 1995; Rao et al., 2004).In several reports, the combination of two fungal bioagents (Aspergillus spp., toxic, and Paecilomyces spp., egg parasitic) was found more effective than a single bioagent against $M$. incognita, resulting in better plant growth (Verma et al., 2009). Parveen et al., (1998) reported the combined efficacy of $P$. aeruginosaand $P$. lilacinus in reducing disease complex caused by the root knot nematode Meloidogyne javanica (Treub) Chitw. and the fungi Macrophomina phaseolina (Tassi) Goid. And Fusarium oxysporum (Synder et Hansen) on pumpkin and F. solani (Mart.) Sacc. on watermelon. Similar results of present investigations of effectiveness of P.lilacinus are in agreement with those reported by Siddiqui et al., (2000) who studied the efficacy of Pseudomonas aeruginosa alone or in combination with Paecilomyces lilacinus against root knot nematode and root infecting fungi under laboratory and field conditions.

The inhibitory effects on egg hatching of root knot nematode due to application of $P$. lilacinus and T.virideis also reported by Goswami and Singh (2004) under laboratory conditions. Paecilo myceslilacinus fungus followed by Pseudomonas fluorescens caused higher rate of juvenile mortality at 1.00 per cent concentration was found to be promising in laboratory bioassay studies and recorded 80.00 per cent mortality of second stage juveniles of root-knot nematode infesting bottle gourd under laboratory conditions(Mane and Mahase, 2017). 
Table.1 Treatment combinations of bionematicide were as under:

\begin{tabular}{|c|c|}
\hline$T_{1}$ & $\begin{array}{c}\text { Purpureocillium lilacinum }\left(\text { cfu } 2 \times 10^{6}\right) @ 2.5 \mathrm{~kg} / \mathrm{ha} \text { along with } 2.5 \text { tons of } \\
\text { Vermicompost } / \mathrm{ha} \text {.......... PI + Vermi }\end{array}$ \\
\hline $\mathbf{T}_{2}$ & $\begin{array}{l}\text { Pochonia chlamydosporia }\left(\mathrm{cfu} 2 \times 10^{6}\right) @ 2.5 \mathrm{~kg} / \mathrm{ha} \text { along with } 2.5 \text { tons of } \\
\text { Vermicompost } / \mathrm{ha} \ldots \ldots . . . \mathrm{Pc}+\text { Vermi }\end{array}$ \\
\hline $\mathbf{T}_{3}$ & $\begin{array}{c}\text { Pseudomonas fluorescens }\left(\mathrm{cfu} 2 \times 10^{6}\right) @ 2.5 \mathrm{~kg} / \mathrm{ha} \text { along with } 2.5 \text { tons of } \\
\text { Vermicompost } / \mathrm{ha} \ldots \ldots \ldots . \mathrm{Pf}+\text { Vermi }\end{array}$ \\
\hline $\mathbf{T}_{4}$ & $\mathrm{~T} 1+\mathrm{T} 3 \quad \ldots \ldots \ldots . \mathrm{Pl}+$ Vermi $+\mathrm{Pf}+$ Vermi \\
\hline$T_{5}$ & $\ldots . \mathrm{Pc}+\mathrm{Vermi}+\mathrm{Pf}+\mathrm{Vermi}$ \\
\hline $\mathbf{T}_{6}$ & 2.5 tons of Vermicompost/ha \\
\hline $\mathbf{T}_{7}$ & Mulching with green leaves of Sunhemp @ $3 \mathrm{~kg} / \mathrm{m}^{2}$ \\
\hline $\mathbf{T}_{8}$ & Bacillus pumilus @ $2.5 \mathrm{~kg}+2.5 \mathrm{t} \mathrm{FYM/ha} \mathrm{...........} \mathrm{Bp} \mathrm{+} \mathrm{sunhemp}$ \\
\hline $\mathbf{T}_{9}$ & Carbofuran $10 \mathrm{~g} / \mathrm{m}^{2}$ \\
\hline$T_{10}$ & Untreated Control \\
\hline
\end{tabular}

\begin{tabular}{|c|c|c|c|c|}
\hline \multicolumn{5}{|c|}{ Table.2 Efficacy of bio-agents in the management of Meloidogyne species in Bitter gourd } \\
\hline \multirow{3}{*}{ Tret. No. } & & & \multicolumn{2}{|c|}{ Year: 2016} \\
\hline & \multicolumn{2}{|c|}{ FNP (Log trans.) } & \multirow{2}{*}{$\begin{array}{c}\text { RKI } \\
(\sqrt{ } \mathbf{x} \text { trans. })\end{array}$} & \multirow{2}{*}{$\begin{array}{c}\text { Yield } \\
\text { t/ha }\end{array}$} \\
\hline & Soil & Root & & \\
\hline $\mathbf{T}_{1}(\mathrm{Pl}+$ Vermi $)$ & $\begin{array}{l}2.784^{\mathrm{de}} \\
(608)\end{array}$ & $\begin{array}{l}2.694^{\mathrm{a}} \\
(494)\end{array}$ & $\begin{array}{l}2.16^{\mathrm{a}} \\
(4.68)\end{array}$ & $5.072^{\mathrm{ab}}$ \\
\hline $\mathbf{T}_{2}(\mathbf{P c}+$ Vermi $)$ & $\begin{array}{l}2.858^{\text {cde }} \\
(721)\end{array}$ & $\begin{array}{l}2.777^{\mathrm{a}} \\
(598)\end{array}$ & $\begin{array}{l}2.21^{\mathrm{a}} \\
(4.88)\end{array}$ & $3.518^{\mathrm{cd}}$ \\
\hline$T_{3}(\mathbf{P f}+$ Vermi $)$ & $\begin{array}{c}2.969^{\mathrm{abc}} \\
(931)\end{array}$ & $\begin{array}{l}2.855^{\mathrm{a}} \\
(716)\end{array}$ & $\begin{array}{l}2.21^{\mathrm{a}} \\
(4.86)\end{array}$ & $3.292^{\text {cde }}$ \\
\hline $\begin{array}{r}\mathrm{T}_{4}(\mathrm{Pl}+\text { Vermi })+ \\
(\mathrm{Pf}+\text { Vermi })\end{array}$ & $\begin{array}{l}2.766^{\mathrm{e}} \\
(583)\end{array}$ & $\begin{array}{l}2.695^{\mathrm{a}} \\
(495)\end{array}$ & $\begin{array}{l}2.17^{\mathrm{a}} \\
(4.72)\end{array}$ & $5.210^{\mathrm{a}}$ \\
\hline $\begin{array}{r}T_{5}(\mathbf{P c}+\text { Vermi }) \\
+(\mathbf{P f}+\text { Vermi })\end{array}$ & $\begin{array}{c}2.838^{\text {cde }} \\
(689)\end{array}$ & $\begin{array}{l}2.721^{\mathrm{a}} \\
(526)\end{array}$ & $\begin{array}{l}2.17^{\mathrm{a}} \\
(4.72)\end{array}$ & $4.421^{\mathrm{abc}}$ \\
\hline $\mathbf{T}_{6}($ Vermi $)$ & $\begin{array}{c}2.933^{\mathrm{abc}} \\
(587)\end{array}$ & $\begin{array}{l}2.821^{\mathrm{a}} \\
(662)\end{array}$ & $\begin{array}{l}2.22^{\mathrm{a}} \\
(4.91)\end{array}$ & $3.468^{\text {cd }}$ \\
\hline $\mathbf{T}_{7}($ Sunhemp) & $\begin{array}{c}2.901^{\mathrm{bcd}} \\
(796)\end{array}$ & $\begin{array}{l}2.775^{\mathrm{a}} \\
(596)\end{array}$ & $\begin{array}{l}2.23^{\mathrm{a}} \\
(4.95)\end{array}$ & $3.498^{\mathrm{cd}}$ \\
\hline $\mathbf{T}_{8}(\mathbf{B p}+$ Sunhemp $)$ & $\begin{array}{l}2.849^{\text {cde }} \\
(706)\end{array}$ & $\begin{array}{l}2.713^{\mathrm{a}} \\
(516)\end{array}$ & $\begin{array}{l}2.22^{\mathrm{a}} \\
(4.93)\end{array}$ & $3.806^{\mathrm{bcd}}$ \\
\hline $\mathbf{T}_{9}(\mathrm{Car})$ & $\begin{array}{l}3.032^{a b} \\
(1076)\end{array}$ & $\begin{array}{l}2.837^{\mathrm{a}} \\
(687)\end{array}$ & $\begin{array}{l}2.24^{\mathrm{a}} \\
(4.99)\end{array}$ & $2.447^{\text {de }}$ \\
\hline $\mathbf{T}_{10}(\mathrm{Con})$ & $\begin{array}{l}3.044^{\mathrm{a}} \\
(1107)\end{array}$ & $\begin{array}{l}2.879^{a} \\
(757)\end{array}$ & $\begin{array}{l}2.19^{\mathrm{a}} \\
(4.81)\end{array}$ & $2.090^{\mathrm{e}}$ \\
\hline S. Em. \pm & 0.04 & 0.1 & 0.03 & 0.41 \\
\hline $\mathrm{CV} \%$ & 2.60 & 6.38 & 2.54 & 19.37 \\
\hline $\begin{array}{l}\text { Figures in parenthes } \\
\text { Figures indicating c } \\
\text { to DNMRT }\end{array}$ & $\begin{array}{l}\text { transfo } \\
\text { etters d }\end{array}$ & $\begin{array}{l}\text { of Lo } \\
\text { signifi }\end{array}$ & $\begin{array}{l}\text { ans } \\
6 \text { lev }\end{array}$ & acce \\
\hline
\end{tabular}




\begin{tabular}{|c|c|c|c|c|}
\hline \multirow{3}{*}{ Tret. No. } & & & \multirow{2}{*}{\multicolumn{2}{|c|}{$\begin{array}{c}\text { Year: } 2017 \\
\text { Yield }\end{array}$}} \\
\hline & \multicolumn{2}{|c|}{$\begin{array}{c}\text { FNP } \\
\text { (Log trans.) }\end{array}$} & & \\
\hline & Soil & Root & $\begin{array}{c}\text { RKI } \\
(\sqrt{ } \mathbf{x} \text { trans. })\end{array}$ & t/ha \\
\hline $\mathrm{T}_{1}(\mathrm{Pl}+$ Vermi $)$ & $\begin{array}{l}2.735^{\mathrm{a}} \\
(543)\end{array}$ & $\begin{array}{c}2.660^{\mathrm{ab}} \\
(457)\end{array}$ & $\begin{array}{l}1.77^{\mathrm{ef}} \\
(3.12)\end{array}$ & $10.433^{\mathrm{a}}$ \\
\hline $\mathbf{T}_{2}(\mathbf{P c}+$ Vermi $)$ & $\begin{array}{l}2.721^{\mathrm{a}} \\
(526)\end{array}$ & $\begin{array}{c}2.662^{\mathrm{ab}} \\
(459)\end{array}$ & $\begin{array}{l}1.98^{\mathrm{bc}} \\
(3.90)\end{array}$ & $8.923^{\mathrm{ab}}$ \\
\hline $\mathbf{T}_{3}(\mathbf{P f}+$ Vermi $)$ & $\begin{array}{l}2.775^{\mathrm{a}} \\
(596)\end{array}$ & $\begin{array}{c}2.623^{a b c} \\
(420)\end{array}$ & $\begin{array}{l}1.93^{\mathrm{bcd}} \\
(3.73)\end{array}$ & $7.493^{\mathrm{bc}}$ \\
\hline $\begin{array}{r}\mathrm{T}_{4}(\mathrm{Pl}+\text { Vermi })+ \\
(\mathrm{Pf}+\text { Vermi })\end{array}$ & $\begin{array}{l}2.706^{\mathrm{a}} \\
(508)\end{array}$ & $\begin{array}{c}2.587^{\mathrm{bc}} \\
(386)\end{array}$ & $\begin{array}{l}1.74^{\mathrm{f}} \\
(3.03)\end{array}$ & $10.483^{\mathrm{a}}$ \\
\hline $\begin{array}{r}\mathrm{T}_{5}(\text { Pc+Vermi }) \\
+(\text { Pf +Vermi })\end{array}$ & $\begin{array}{l}2.760^{\mathrm{a}} \\
(575)\end{array}$ & $\begin{array}{c}2.607^{\mathrm{abc}} \\
(404)\end{array}$ & $\begin{array}{l}1.84^{\mathrm{de}} \\
(3.37)\end{array}$ & $9.428^{\mathrm{ab}}$ \\
\hline $\mathbf{T}_{6}($ Vermi $)$ & $\begin{array}{l}2.768^{\mathrm{a}} \\
(586)\end{array}$ & $\begin{array}{l}2.670^{\mathrm{ab}} \\
(468)\end{array}$ & $\begin{array}{l}1.90^{\mathrm{cd}} \\
(3.62)\end{array}$ & $8.800^{\mathrm{ab}}$ \\
\hline $\mathbf{T}_{7}$ (Sunhemp) & $\begin{array}{l}2.899^{\mathrm{a}} \\
(792)\end{array}$ & $\begin{array}{l}2.679^{a b} \\
(477)\end{array}$ & $\begin{array}{l}2.16^{\mathrm{a}} \\
(4.68)\end{array}$ & $8.476^{\mathrm{abc}}$ \\
\hline $\mathbf{T}_{8}(\mathbf{B p}+$ Sunhemp $)$ & $\begin{array}{l}2.704^{\mathrm{a}} \\
(506)\end{array}$ & $\begin{array}{l}2.558^{\mathrm{c}} \\
(361)\end{array}$ & $\begin{array}{l}1.84^{\mathrm{de}} \\
(3.37)\end{array}$ & $8.967^{\mathrm{ab}}$ \\
\hline $\mathrm{T}_{9}$ (Car) & $\begin{array}{l}2.877^{\mathrm{a}} \\
(753)\end{array}$ & $\begin{array}{c}2.679 \mathrm{ab} \\
(477)\end{array}$ & $\begin{array}{l}2.01^{\mathrm{b}} \\
(4.02)\end{array}$ & $7.223^{b c}$ \\
\hline $\mathbf{T}_{10}(\mathrm{Con})$ & $\begin{array}{l}2.938^{\mathrm{a}} \\
(867)\end{array}$ & $\begin{array}{l}2.694^{\mathrm{a}} \\
(494)\end{array}$ & $\begin{array}{l}2.20^{\mathrm{a}} \\
(4.02)\end{array}$ & $6.436^{\mathrm{c}}$ \\
\hline S. Em. \pm & 0.07 & 0.03 & 0.03 & 0.70 \\
\hline $\mathrm{CV} \%$ & 4.40 & 1.87 & 2.56 & 14.01 \\
\hline
\end{tabular}

Figures in parentheses are retransformed values of Log and $\sqrt{x}$ trans

Figures indicating common letters do not differ significantly at 5\% level of significance according to DNMRT 
Table.4 Efficacy of bio-agents in the management of Meloidogyne species in Bitter gourd

\begin{tabular}{|c|c|c|c|c|}
\hline & & & \multicolumn{2}{|c|}{ Year: 2018} \\
\hline \multirow[t]{2}{*}{ Tret. No. } & \multicolumn{2}{|c|}{$\begin{array}{c}\text { FNP } \\
\text { (Log trans.) }\end{array}$} & \multirow[t]{2}{*}{$\begin{array}{c}\text { RKI } \\
(\sqrt{ } \mathbf{x} \text { trans. })\end{array}$} & Yield \\
\hline & Soil & Root & & t/ha \\
\hline $\mathbf{T}_{1}(\mathrm{Pl}+$ Vermi $)$ & $\begin{array}{l}2.021^{f} \\
(105)\end{array}$ & $\begin{array}{c}2.597^{\mathrm{bc}} \\
(396)\end{array}$ & $\begin{array}{l}1.71^{\mathrm{a}} \\
(3.07)\end{array}$ & $7.31^{\mathrm{a}}$ \\
\hline $\mathbf{T}_{2}(\mathbf{P c}+$ Vermi $)$ & $\begin{array}{l}2.321^{\mathrm{d}} \\
(210)\end{array}$ & $\begin{array}{c}2.632^{\mathrm{abc}} \\
(432)\end{array}$ & $\begin{array}{l}1.88^{\mathrm{a}} \\
(3.80)\end{array}$ & $3.82^{\mathrm{cd}}$ \\
\hline $\mathbf{T}_{3}(\mathbf{P f}+$ Vermi $)$ & $\begin{array}{l}2.618^{c} \\
(415)\end{array}$ & $\begin{array}{c}2.677^{\mathrm{ab}} \\
(475)\end{array}$ & $\begin{array}{l}1.98^{\mathrm{a}} \\
(4.00)\end{array}$ & $2.26^{\mathrm{e}}$ \\
\hline $\begin{array}{r}\mathrm{T}_{4}(\mathrm{Pl}+\text { Vermi })+ \\
(\mathrm{Pf}+\text { Vermi })\end{array}$ & $\begin{array}{c}1.977^{\mathrm{f}} \\
(95)\end{array}$ & $\begin{array}{l}2.566^{c} \\
(375)\end{array}$ & $\begin{array}{l}1.60^{\mathrm{a}} \\
(2.73)\end{array}$ & $8.38^{\mathrm{a}}$ \\
\hline $\begin{array}{r}\mathrm{T}_{5}(\text { Pc+Vermi }) \\
+(\text { Pf }+ \text { Vermi })\end{array}$ & $\begin{array}{l}2.381^{\mathrm{d}} \\
(241)\end{array}$ & $\begin{array}{c}2.667^{\mathrm{ab}} \\
(465)\end{array}$ & $\begin{array}{l}1.96^{\mathrm{a}} \\
(3.88)\end{array}$ & $5.84^{b}$ \\
\hline$T_{6}($ Vermi $)$ & $\begin{array}{l}2.581^{\mathrm{c}} \\
(384)\end{array}$ & $\begin{array}{c}2.668^{\mathrm{ab}} \\
(472)\end{array}$ & $\begin{array}{l}1.96^{\mathrm{a}} \\
(4.00)\end{array}$ & $2.86^{\mathrm{de}}$ \\
\hline $\mathbf{T}_{7}$ (Sunhemp) & $\begin{array}{c}2.844^{b} \\
(698)\end{array}$ & $\begin{array}{c}2.685^{\mathrm{ab}} \\
(488)\end{array}$ & $\begin{array}{l}2.07^{\mathrm{a}} \\
(4.27)\end{array}$ & $2.72^{\mathrm{de}}$ \\
\hline $\mathbf{T}_{8}(\mathbf{B p}+$ Sunhemp $)$ & $\begin{array}{l}2.229^{\mathrm{e}} \\
(170)\end{array}$ & $\begin{array}{c}2.618^{a b c} \\
(418)\end{array}$ & $\begin{array}{l}1.80^{\mathrm{a}} \\
(3.40)\end{array}$ & $4.65^{\mathrm{bc}}$ \\
\hline $\mathrm{T}_{9}(\mathrm{Car})$ & $\begin{array}{l}3.027^{\mathrm{a}} \\
(1064)\end{array}$ & $\begin{array}{c}2.685^{\mathrm{ab}} \\
(486)\end{array}$ & $\begin{array}{l}2.07^{\mathrm{a}} \\
(4.33)\end{array}$ & $2.04^{\mathrm{e}}$ \\
\hline $\mathbf{T}_{10}($ Con $)$ & $\begin{array}{l}3.067^{\mathrm{a}} \\
(1166)\end{array}$ & $\begin{array}{l}2.703^{\mathrm{a}} \\
(507)\end{array}$ & $2.15^{\mathrm{a}}(4.67)$ & $1.82^{\mathrm{e}}$ \\
\hline S. Em. \pm & 0.02 & 0.03 & --- & 4.19 \\
\hline CV \% & 1.22 & 1.83 & 1.67 & 17.41 \\
\hline
\end{tabular}

Figures in parentheses are retransformed valuesof Log and $\sqrt{x t r a n s}$

Figures indicating common letters do not differ significantly at 5\% level of significance according to DNMRT 


\begin{tabular}{|c|c|c|c|c|c|}
\hline \multirow{3}{*}{ Tret. No. } & & & Pool & 3 years & 2018) \\
\hline & \multicolumn{2}{|c|}{$\begin{array}{c}\text { FNP } \\
\text { (Log trans.) }\end{array}$} & \multirow[t]{2}{*}{$\begin{array}{c}\text { RKI } \\
(\sqrt{ } \mathrm{x} \text { trans. })\end{array}$} & \multirow{2}{*}{ Yield } & \multirow[t]{2}{*}{ ICBR } \\
\hline & Soil & Root & & & \\
\hline $\mathrm{T}_{1}(\mathrm{Pl}+$ Vermi $)$ & $\begin{array}{r}2.513^{\mathrm{d}} \\
(428)\end{array}$ & $\begin{array}{c}2.651^{\mathrm{bcd}} \\
(472)\end{array}$ & $\begin{array}{l}1.88^{\mathrm{bc}} \\
(3.63)\end{array}$ & $7.60^{\mathrm{a}}$ & $1: 5.40$ \\
\hline$T_{2}(\mathbf{P c}+$ Vermi) & $\begin{array}{c}2.633^{\mathrm{cd}} \\
(486)\end{array}$ & $\begin{array}{c}2.690^{\text {abcd }} \\
(501)\end{array}$ & $\begin{array}{c}2.02^{\mathrm{abc}} \\
(4.20)\end{array}$ & $5.42^{\mathrm{bc}}$ & $1: 2.03$ \\
\hline $\mathbf{T}_{3}(\mathbf{P f}+$ Vermi $)$ & $\begin{array}{c}2.787^{\mathrm{abcd}} \\
(690)\end{array}$ & $\begin{array}{c}2.718^{a b c} \\
(539)\end{array}$ & $\begin{array}{l}2.04^{\mathrm{ab}} \\
(4.20)\end{array}$ & $4.35^{\text {cde }}$ & $1: 0.38$ \\
\hline $\begin{array}{r}\mathrm{T}_{4}(\mathrm{PI}+\text { Vermi })+ \\
(\mathrm{Pf}+\text { Vermi })\end{array}$ & $\begin{array}{r}2.483^{\mathrm{d}} \\
(401)\end{array}$ & $\begin{array}{r}2.616^{\mathrm{d}} \\
(436)\end{array}$ & $\begin{array}{l}1.84^{\mathrm{c}} \\
(3.50)\end{array}$ & $8.02^{\mathrm{a}}$ & $1: 2.55$ \\
\hline $\begin{array}{r}\mathrm{T}_{5}(\mathrm{Pc}+\text { Vermi }) \\
+(\mathbf{P f}+\text { Vermi })\end{array}$ & $\begin{array}{l}2.660^{\mathrm{bcd}} \\
(504)\end{array}$ & $\begin{array}{c}2.665^{\text {abcd }} \\
(480)\end{array}$ & $\begin{array}{r}2.00^{\mathrm{abc}} \\
(3.99)\end{array}$ & $6.56^{\mathrm{ab}}$ & $1: 1.42$ \\
\hline$T_{6}($ Vermi) & $\begin{array}{c}2.766^{\text {abcd }} \\
(621)\end{array}$ & $\begin{array}{c}2.719^{\mathrm{abc}} \\
(537)\end{array}$ & $\begin{array}{c}2.02^{\mathrm{abc}} \\
(4.18)\end{array}$ & $5.04^{\mathrm{cd}}$ & $1: 1.51$ \\
\hline $\mathbf{T}_{7}$ (Sunhemp) & $\begin{array}{c}2.882^{\mathrm{abc}} \\
(769)\end{array}$ & $\begin{array}{c}2.713^{\mathrm{abc}} \\
(522)\end{array}$ & $\begin{array}{l}2.15^{\mathrm{a}} \\
(4.64)\end{array}$ & $4.90^{\mathrm{cd}}$ & -- \\
\hline $\mathbf{T}_{8}(\mathbf{B p}+$ Sunhemp $)$ & $\begin{array}{l}2.594^{\text {cd }} \\
(463)\end{array}$ & $\begin{array}{c}2.630^{\text {cd }} \\
(457)\end{array}$ & $\begin{array}{l}1.95^{\mathrm{bc}} \\
(3.90)\end{array}$ & $5.80^{\mathrm{bc}}$ & $1: 10.15$ \\
\hline$T_{9}($ Car $)$ & $\begin{array}{c}2.979^{\mathrm{ab}} \\
(969)\end{array}$ & $\begin{array}{c}2.734^{\mathrm{ab}} \\
(554)\end{array}$ & $\begin{array}{r}2.10^{\mathrm{abc}} \\
(4.45)\end{array}$ & $3.90^{\mathrm{de}}$ & -- \\
\hline$T_{10}($ Con) & $\begin{array}{l}3.016^{\mathrm{a}} \\
(1048)\end{array}$ & $\begin{array}{c}2.759^{\mathrm{a}} \\
(593)\end{array}$ & $\begin{array}{l}2.18^{\mathrm{a}} \\
(4.78)\end{array}$ & $3.45^{\mathrm{e}}$ & \\
\hline \multicolumn{6}{|l|}{ Treatment } \\
\hline S. Em. \pm & 0.10 & 0.03 & 0.06 & 4.51 & \\
\hline $\mathbf{Y} \times \mathbf{T}$ & 0.139 & NS & NS & 1.49 & \\
\hline CV \% & 3.11 & 1.83 & 9.43 & 16.61 & \\
\hline
\end{tabular}

Figures in parentheses are retransformed valuesof Log and $\sqrt{x}$ trans

Figures indicating common letters do not differ significantly at 5\% level of significance according to DNMRT

Biological activity of BioAct WG (Paecilomyces lilacinus strain 251) and Trichoderma viride strain T6 applied alone and in combination against root-knot nematodes (Meloidogyne spp.) in cucumber were effective in management of root-knot nematodes (Meloidogyne spp.) in cucumbers (VinelinaYankova et al., 2014).

Hence, the use of a combination formulation of $P$. lilacinum and $P$. fluorescens enriched in vermicompost would be very useful for the management of the nematode induced disease in bitter gourd. Therefore, this formulation could be proposed as an ideal component of an integrated pest management package.

\section{References}

Ehteshamul-Haque S., Abid M. and Ghaffar A., 1995. Efficacy of Bradyrhizobium sp., and Paecilomyces lilacinus with oil cakes in the control of root rot of mungbean. 
Tropical Science, 35: 294-299.

Goswami, B.K. and Singh, S., 2004. Fungal bioagents for management of root-knot nematode in tomato. Pesticide Res. J., 16 (1) : 9-12.

Izhar I., Ehteshamul-Haque S., Javeed M. and Ghaffar A., 1995. Efficacy of Pseudomonas aeruginosa and Bradyrhizobium sp. in the control of root rot disease in chickpea. Pakistan Journal of Botany, 27: 451-455.

Jain, R.K., Mathur, K.N. and Singh, R.V., 2007. Estimation of losses due to plant parasitic nematodes on different crops in India. Indian J. Nematol., 37: 23-25.

Mane, P.B. and Mhase, N. L., 2017.Bioefficacy of different bioagents against root-knot nematode, Meloidogyne incognita infesting bottle gourd under laboratory conditions. International Journal of Plant Protection, 10(1): 87-91.

Parveen S., EhteshamulHaque S. and Ghaffar A., 1998. Efficacy of Pseudomonas aeruginosa and Paecilomyces lilacinus in the control of root rot-root knot disease complex on some vegetables. Nematologia Mediterranea, 26: 209-212.

Rao M.S., DhananjayNaik and Shylaja M.,
2004.Bio-intensive management of rootknot nematodes on bell pepper using Pochonia chlamydosporia and Pseudomonas fluorescens. Nematologia Mediterranea, 32: 159-163.

Sasser, J.N. and Freckman, D.W., 1987.World perspective on Nematology. The role of the society. pp 7-14. In: Vistas of nematology. Society of Nematologists, Hyattsville, Maryland, U.S.A.

Siddiqui, I.A., Qureshi, S.A., Sultana, V., Haque, E.S. and Ghaffar, A., 2000. Biological control of root rot- root knot disease complex of tomato. Plant \& Soil., 22(7): 163-169.

Verma K.K., Goel S.R. and Nandal S.N., 2009. Efficacy of fungal antagonist as seed treatment in the management of Meloidogyne javanica in cowpea. Indian Journal of Nematology, 39: 198-2000.

Yankova, V, Markova, D, Naidenov, B, Arnaoudov, B., 2014. Management of Root-Knot Nematodes (Meloidogyne spp.) in Greenhouse Cucumbers Using Microbial Products. Turkish Journal of Agricultural and Natural Sciences (2) 15669-1573.

\section{How to cite this article:}

Tulika Singh, A. D. Patel, Anjana Prajapati and Patel, B. A. 2019. Biomanagement of Meloidogyne Species in Bitter Gourd. Int.J.Curr.Microbiol.App.Sci. 8(10): 546-553. doi: https://doi.org/10.20546/ijcmas.2019.810.059 